\title{
Oxidative stress in children chronic hepatitis
}

\author{
Alice N. Azoicai, Bogdan A. Stana \\ $2^{\text {nd }}$ Clinic of Pediatrics, "Sf. Maria" Emergency Hospital for Children, I"Gr. T. Popa" \\ University of Medicine and Pharmacy, Iasi, Romania
}

\begin{abstract}
Oxidative stress reduces the efficacy of the immune response effector mechanisms, making the cells more susceptible to apoptosis. In lymphoid cells, free radicals interfere with the response to antiviral treatment, causing resistance to therapy and favoring chronic infection. One of the factors responsible for oxidative stress in chronic infection with hepatitis $B$ is the production of pro-inflammatory cytokines. On the other hand, studies show that $\mathrm{C}$ hepatitis virus can directly induce oxidative stress in hepatocytes. Chronic $\mathrm{C}$ hepatitis core gene expression was associated with elevated reactive oxygen species markers, decreased intracellular and/or mitochondrial glutation content and increased level of oxidized thioredoxin and lipid peroxidation products. Moreover, increased level of the malonyl-dialdehyde in serum of patients with hepatocarcinoma is suggesting that MDA can be a reliable marker of liver injury quantification.

Reduction of the oxidative stress can be done using antioxidant medication (hepatoprotectives, ursodeoxycholic acid and vitamin A, C, E), this approach representing an important benefit for the patient.
\end{abstract}

Keywords: oxidative stress, chronic hepatitis, child

Oxidative stress affects the action of effector mechanisms of the immune response, making the cells more susceptible to apoptosis. On lymphoid cells, free radicals interfere with the response to antiviral treatment, causing resistance to therapy and favoring chronic infection. Hepatitis B and C occur in amplifying oxidative stress in chronic infection through different mechanisms.

There are some different mechanisms of oxidative stress amplification regarding $B$ and $C$ hepatitis viruses. One of the factors responsible for oxidative stress in chronic infection with hepatitis B is increased production of pro-inflammatory cytokines. In these patients were detected elevated levels of TNF $\alpha$, responsible for increasing the rate of production of superoxide in mitochondria. Also, in patients with chronic hepatitis B (HBV) authors objectified a decreased activity of glutathione (GST), directly proportional to the rate of progression to hepatocellular carcinoma (HCC). A large part of patients with chronic hepatitis $\mathrm{C}(\mathrm{HCV})$, in which oxidative stress manifests itself strongly, leading to resistance therapy, have also well repre- sented deposits of cellular iron, iron serving as substrate for activation of the cascade of oxidative stress. (1)

\section{The mechanisms of oxidative stress in HBV infection}

Reactive oxygen species and lipid peroxidation products can contribute both to the installation and progression of hepatic fibrosis. In addition, oxidative stress directly affects the viral DNA, which can lead to the development of hepatocellular carcinoma (HCC).

Cengiz et al. have evaluated oxidative status of those patients mainly by measuring the total antioxidant response (TAR) of subjects with chronic HBV with or without cirrhosis. (2) It was measured also the total peroxide level for the same plasma samples. The ratio between the total plasma peroxide levels in the plasma versus TAR value was defined as oxidative stress index. There were $33 \mathrm{sub}-$ jects included in the study, 20 males and 13 females, with an average age of $39 \pm 12.8$ years. Subjects with HVB cirrhosis $(n=12)$ were 7 males and 5 fe- 
males, mean age $40.9 \pm 11.6$ years. The control group included 16 subjects, 9 males and 7 females, mean age $31.6 \pm 6$ years. Results showed that the total antioxidant response (TAR) was significantly lower in patients with cirrhosis compared to the control group. The difference between HVB subjects and subjects with cirrhosis related to HVB infection, in terms of TAR, was not statistically significant. Still, the total peroxide plasma of subjects with HBV versus those with cirrhosis was significantly higher than in the control group. Regarding the relationship with the biological findings versus TAR, the authors couldn't find a significant correlation with the ALT values, which are the most frequent markers of active infection. However, serum ALT levels were positively correlated with the total level of peroxide in subjects with chronic hepatitis B. (2)

Tissue damage due to oxidative stress in chronic hepatitis B is accompanied by a significant increased levels of fibrogenic cytokines in plasma, $\mathrm{TGF} \beta, \mathrm{TNF} \alpha$, respectively. In addition, reactive oxygen species accumulated in large quantities in the inflamed tissue can cause injury to the host cells and also can induce DNA damage with the appearance of new mutations. DNA damage under the action of oxidative stress plays an important role in the development of liver cirrhosis, with later-on progression of chronic B infection to $\mathrm{HCC}$, as proven by studies undertaken.

Quantification of aflatoxin B (AFB1) levels, the exposure to oxidative stress biomarkers and modification of viral replication were assessed in patients with hepatocellular carcinoma (HCC). The plasma level of AFB1 was significantly higher than in the control group. (3) This survey indicates a significant contribution of HBV in oxidative stress progression in a population exposed to AFB1, which can substantially increase the risk of evolving towards HCC.

Pre-S mutants can influence and modulate the activity of COX-2 by the p38 MAPK and NFkB pathways. The excess of COX-2 production was detected in many types of cancer and it is linked to the progression of the disease. Experimental models (in transgenic mice) expressing pre-S protein mutants showed higher levels of COX-2 in tissue samples taken from liver and kidney. Similarly, increased expression of COX-2 has been observed in biopsied human tissues taken from individuals with chronic B hepatitis and HCC.

In order to assess the effect of pre-S mutants and oxidative stress in the endoplasmic reticulum (ER) and the evolution towards chronic infection, cirrhosis and HCC, Hui-Ching Wang et al. performed a cohort study on several patients with HBV infection. (4). Pre-S mutant proteins are accumulated in ER and induce ER stress signals (Fig. 1). Colorimetric methods using a dual-labeled fluorescence microscopy revealed substantial agglomeration of pre-S protein mutants and the ER calregulin, which suggests that these mutant proteins, LHBs $\Delta \mathrm{S} 1$, and $\triangle \mathrm{S} 2$-LHBs can accumulate in the ER. In natural history, the accumulation of pre-S mutants in ER can induce subsequent signals leading to cell degeneration and progression to HCC. Also, pre-S proteins of mutant genes induce genomic instability, which can encourage the action of oxidative stress, thereby producing DNA damage with increasing frequency of new mutations.

To demonstrate that HBV patients with increased expression of the protein pre-S mutants carry a higher risk of developing HCC, authors conducted a prospective study in a group of pat HBs Ag positive patients. They studied 68 men with newly diagnosed HCC and 132 subjects in the control group without HCC with HBV infection. Among these cases, 14 men with HCC (20.6\%) and 11 in the control group $(8.3 \%)$ had detectable mutant pre-S protein in the plasma samples. On a follow-up of 10 years, patients who developed pre-S mutants had a higher risk of developing $\mathrm{HCC}$ than other subjects. (HI Yang and Chen CJ, unpublished data). These data indicate that the occurrence of pre-S mutants may present in a higher risk of developing $\mathrm{HCC}$ in patients with chronic HBV infection.

Considering the results of studies presented above, oxidative stress can play a critical role in determining and progression of liver injury, associated with disease severity and with the status of virus replication in chronic B infection. Thus, it requires assessing the potential therapeutic lines targeting this pathogenic pathway.

\section{The mechanisms of oxidative stress in HCV infection}

The application of eligibility criteria for antiviral treatment incited studying the role of oxidative stress mechanisms of action (2). These injuries are induced by various processes, including:

- Activation of NAD(P)H oxidase in Kupffer cells and polymorphonuclear cells during inflammation;

- Iron overload and lipid peroxidation;

- Activation of NAD(P)H oxidase by the NS3 protein;

- Increase mitochondrial ROS production/ SRN by the electron transport chain bound to proteins NS5A and core; 


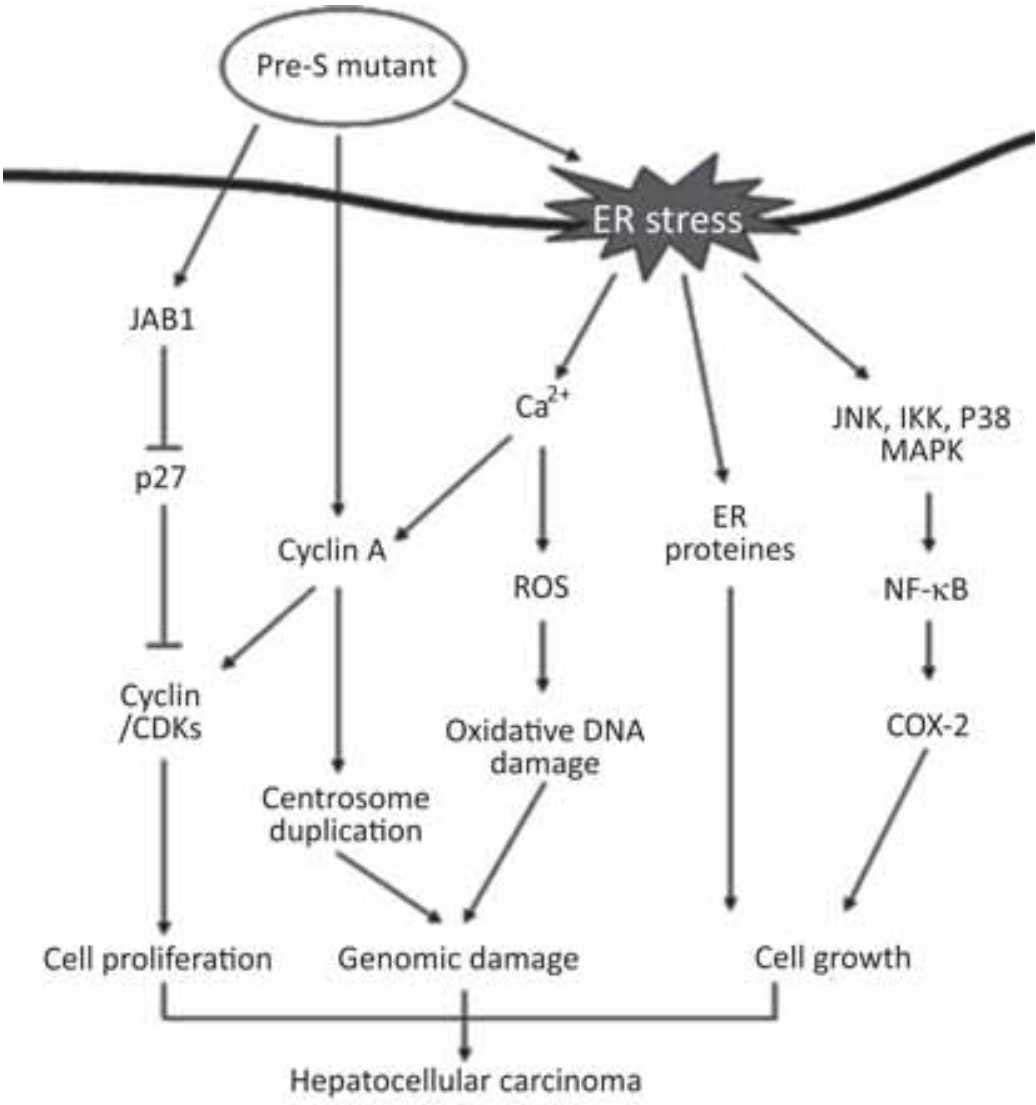

FIGURE 1. Oncogenic pathway of hepatitis $B$ virus pre-S mutant proteins. Adapted from Hui-Ching Wang, Wenya Huang, Ming-Der Lai, Ih-Jen Su, Hepatitis B virus pre-S mutants, endoplasmic reticulum stress and hepatocarcinogenesis Cancer Science, Volume 97, Issue 8, August 2006 Pages, 683-688 (CDK, cyclin dependent kinase; COX-2, cyclooxygenase-2; ER, endoplasmic reticulum; IKK, Ikappa B kinase; JAB1, Jun activation domain-binding protein 1; JNK, Jun N-terminal kinase; MAPK, mitogen-activated protein kinase; $N K \kappa B$, nuclear factor $K B$; ROS-reactive oxygen species)
- Reduced production of GSH due to liver damage;

- Decreased amount of antioxidants in cells and reducing their gene expression;

- Alcohol, drugs, other toxic elements;

- Increasing cytokines and SRO augmentation;

- Increased CYP2E1 expression;

- Increased expression/activity of Cox-2.

On the other hand, studies show that chronic $\mathrm{HCV}$ can directly induce oxidative stress in hepatocytes. HCV core gene expression was associated with increased SRO, decreased intracellular and/or mitochondrial GSH content and increased oxidized thioredoxin and lipid peroxidation products.

Chronic $\mathrm{C}$ hepatitis infection progresses frequently to cirrhosis, liver failure or hepatocellular carcinoma. To elucidate the role of oxidative stress regarding unfavorable evolution of these patients authors assessed blood levels and / or urinary markers of lipid peroxidation, together with selenium and vitamins A, C, E in a group of 42 patients with hepatitis $\mathrm{C}$ infection. Also, the authors assessed the degree of liver fibrosis in a subset of 33 patients. (6)

The results showed that markers of lipid peroxidation (mainly 8-isoprostane) were elevated in serum of patients, and the level of reduced glutathione significantly decreased in mitochondria. Antioxi- dants (glutathione, selenium and vitamins A, C and E) have decreased compared to age and sex of subjects in the control group. The values were much lower for cirrhotic subjects, but significant changes were observed also in the non-cirrhotic group. Fibrosis score was positively correlated with urinary values d 8-isoprostane, and negatively with the level of vitamin A. In conclusion, as reflected in the results above, oxidative stress is an important feature of infection with hepatitis C. Although antioxidants have been marked as declined products more severe in cirrhotic patients group, there is a clear evidence of the influence of oxidative stress action also in non-cirrhotic patients. Therefore, antioxidant therapy may have a role in slowing the progression of the chronic $\mathrm{C}$ infection to cirrhosis or malignant degeneration.

Indirect evidence suggests that oxidative stress and injury to the mitochondria plays an important role in the unfavorable evolution of chronic $\mathrm{C}$ infection. Some studies have tried to determine if $\mathrm{HCV}$ core protein alters mitochondrial function, thereby contributing to the initiation and maintenance of oxidative stress (7). Thus, the HCV core protein was quantified by its expression in three different cell lines, as well as reactive oxygen species (ROS) and lipid peroxidation products. Core protein levels increased in two cell lines; also, the 
products of lipid peroxidation and antioxidant gene expression have risen significantly. A sequence of the core protein expressed on the mitochondrial cytochrome $\mathrm{C}$ was associated with a redistribution in the cytosol, thus proving oxidative stress damage at this level. The cell sensitivity to oxidative stress was observed in the transgenic mice infected with $\mathrm{HCV}$ who have registered an increase in intra-hepatic lipid peroxidation, as response to the action of carbon tetrachloride. Study findings focused on oxidative damage that occurred as a direct result of $\mathrm{HCV}$ core protein expression, both in vitro and in vivo, and can exert a direct cytopathic effect on mitochondria. These results provide a new perspective in the pathogenesis of hepatitis $\mathrm{C}$, providing a rationale for investigating antioxidant therapy as adjunctive treatment in these patients.

Chronic infection with $\mathrm{HCV}$ is carcinogenic (not only in the liver tissue, but also in lymphoid tissue), an effect which correlates with the decrease of GST activity, suggesting its important role in determining the cytopathic effect of HCV. Moreover, there were detected increased levels of the malonyl-dialdehyde in serum of patients with $\mathrm{HCC}$, suggesting as a reliable marker of liver injury the quantification of alpha-GST, as it can be found in high concentrations within hepatocytes. Alpha-GST levels in the blood increases rapidly at the time of injury and fall down just as quickly when the aggression stops. Thus, alpha-GST may be the most sensitive marker of response to antiviral therapy. Also, administration of $\mathrm{N}$-acetylcysteine concurrently with interferon leads to sustained therapeutic response in patients with hepatocellular carcinoma. $(8,9)$

In support of these allegations we mention Görenek L et al (2006) study on a group of 19 patients with chronic HCV infection and a control group of 28 healthy individuals. In both groups authors dosed the serum levels of alanine aminotransferase (ALT) and aspartate aminotransferase (AST), and the level of malonyl-dialdehyde (MDA) and superoxide dismutase (SOD), glutathione peroxidase of red blood cells. The measurements were carried out before and after antiviral treatment with interferon alfa-2b and ribavirin for 48 weeks. (10)

Serum levels of MDA significantly increased in patients with chronic HCV prior to treatment, compared to healthy subjects. Also, the concentration of MDA significantly decreased after treatment, as well as the ALT and AST, in this group of patients. Antioxidant enzymes (superoxide dismutase and glutathione peroxidase) had significantly lower levels in erythrocytes of patients with chronic hep- atitis $\mathrm{C}$ before treatment compared to the control group. In conclusion, the results show that patients with chronic $\mathrm{HCV}$ infection are under the influence of oxidative stress, its action being associated with lower levels of antioxidant enzymes. Although interferon and ribavirin, as anti-viral agents, have antioxidant capacity in their action of reducing viral replication and reducing inflammation, perhaps, by this mechanism, they could also decrease the intensity of virus-induced oxidative stress.

Thus, reduction of the oxidative stress medication can be done using antioxidant (hepatoprotectors, ursodeoxycholic acid and vitamin A, C, E), this approach representing an important benefit for the patient.

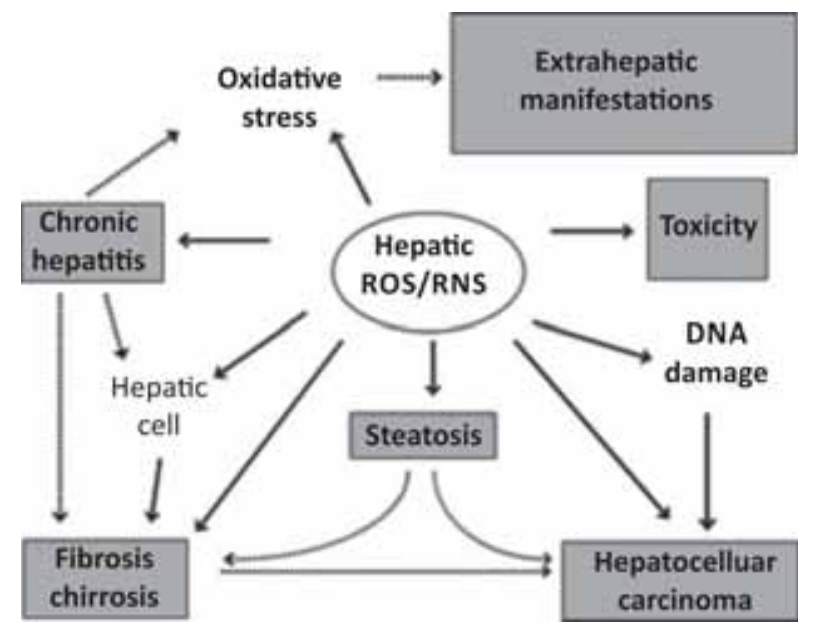

FIGURE 2. Biologic effects of ROS/RNS in chronic C hepatitis (adapted after Choi, J, Ou JHJ, Mechanisms of Liver Injury. III. Oxidative stress in the pathogenesis of hepatitis $C$ virus, Am J of Phys, May 2006, 290(5), 847-851)

The presence of oxidative stress, with elevated serum levels of malonyl-dialdehyde (MDA), a prooxidative marker and significantly decreased serum levels of glutathione (an endogenous antioxidant factor) is proven in chronic hepatitis $\mathrm{C}$ with extrahepatic manifestations in adults but also in chronic hepatitis B in pediatric patients. There is also a direct correlation between serum levels of MDA, GSH and the transferases level in acute hepatitis $\mathrm{C}$ virus, in contrast to chronic infection, where the correlation is only between MDA and serum transaminases, suggesting that serum levels of MDA and GSH are not interrelated with the degree of hepatocyte necrosis (11). Correlation between the HCV-RNA levels and serum levels of MDA and GSH nor in acute hepatitis, or in chronic hepatitis $\mathrm{C}$, was not evidenced, meaning that the viremia level cannot faithfully reflect the value of oxidative stress in blood.

Laboratory data showed that the type of the GSTM1 0 can be correlated with more severe evo- 
lution of $\mathrm{HCV}$ infection, with a positive predictive value of $80 \%$ to chronicity. Determination of HLA incriminated in chronic $\mathrm{HCV}$ infection for patients with type 0 GSTM1, knowing the role in the evolution of some of them to severe HCV infection and to potentiate the extrahepatic manifestations, is an important marker in these patients follow-up. $(11,12)$

Oxidative stress parameters have significantly altered values in pediatric patients with chronic hepatitis $\mathrm{C}$ versus those with hepatitis B. Although interferon and ribavirin are not antioxidants, for their ability to inhibit viral replication, they can reduce inflammation, and thus evolution towards fibrosis, relieving oxidative stress. The positive results obtained from antiviral therapy, although viral load was high, due to the young age of patients and the precocity of treatment.

The presence of extrahepatic manifestations does not change the level of oxidative stress markers. GSTM1 predominance of type 0 is demonstrated in relatively equal percentages both in patients with chronic $\mathrm{HCV}$ infection without extrahepatic manifestations, and those who associate these symptoms. (11) Thus, serum malonyl-dialdehyde, reduced glutathione and glutathione $\mathrm{S}$ transferase serum can be used as biomarkers of the severity and evolution of hepatitis $C$ virus infection.

Recent studies have focused on the pathogenesis of hepatitis infections, showing that excess production of reactive oxygen species (ROS) is involved in the pathogenesis of hepatitis C. (15) Studies assessed the plasma antioxidant status of patients infected with HCV, both those who received standard therapy and those without antiviral treatment. The measurements were carried out before and after supplementation with vitamins $\mathrm{E}, \mathrm{C}$ and zinc (as anti-oxidants). Oxidative stress biomarkers were evaluated in three groups of patients: group $1-$ control; group 2 - patients with HCV without treat-

\section{REFERENCES}

1. Moriya K., Nakagawa K., Santa T., Shintani Y., et al. Oxidative stress in the absence of inflammation in a mouse model for hepatitis C virus, Cancer Res (2001) 61: 4365-70

2. Cengiz Bolukbas, Fusun Filiz Bolukbas, Mehmet Horoz, Mehmet Aslan, Hakim Celik and Ozcan Erel, Increased oxidative stress associated with the severity of the liver disease in various forms of hepatitis B virus infection, BMC Infectious Diseases20055:95,DOI: 10.1186/1471-2334-5-95)

3. Zhi-Ming Liua, Le-Qun Lia, Min-Hao Penga, Tang-Wei Liua, Zhong Qina, Ya Guoa, Kai-Yin Xiaoa, Xin-Ping Yea, et al. Hepatitis B virus infection contributes to oxidative stress in a population exposed to aflatoxin B1 and high-risk for hepatocellular carcinoma, Cancer Letters, Volume 263, Issue 2, Pages 212-222 ment before and after daily supplementation with antioxidants (vitamin E $800 \mathrm{mg}$, Vitamin C $500 \mathrm{mg}$, Zinc $40 \mathrm{mg}$ ) for 6 months; group 3 included HCV patients treated with pegylated interferon and ribavirin. These patients in group 3 were also investigated before and after the same daily antioxidant therapy. Prior to antiviral therapy with interferon and ribavirin, HCV patients had higher levels of superoxide dismutase, the activities of superoxide catalase, and glutathione peroxidase being elevated; they showed a decrease in the activity of glutathione reductase, while the products of lipid peroxidation was raised and glutathione with a lower level compared to the control group. Treatment with standard therapy intensified activities of enzymes (catalase, glutathione S-transferase), increased protein content (carbonyl), leading ultimately to the depletion of glutathione quantities. After supplementation with anti-oxidants, both catalase and glutathione S-transferase activities decreased in patients in group 2, along with lipid peroxidation. Meanwhile, AST, ALT and GGT showed significant increases in the same patients from lot 2 before antioxidant therapy. Ultimately, patients with $\mathrm{HCV}$, whether or not they receive standard antiviral therapy, are at risk of developing irreversible progression to cirrhosis and/or hepatocellular carcinoma in virus-induced oxidative stress conditions. Antioxidant agent supplementation confers efficient protection for both groups, by reducing oxidation processes related illness.

In conclusion, both chronic hepatitis $\mathrm{B}$ virus infection and the virus $\mathrm{C}$ involve tissue damage by direct oxidative stress in hepatocyte. Pathogenic pathways follow different mechanisms of action, which ultimately lead to malignant degeneration. Evidence of studies presented above requires therapeutic approaches to reduce oxidative stress, associated with standard antiviral therapy.

Conflict of interest: none declared Financial support: none declared

4. Hui-Ching Wang, Wenya Huang, Ming-Der Lai, Ih-Jen Su, Hepatitis $B$ virus pre-S mutants, endoplasmic reticulum stress and hepatocarcinogenesis Cancer Science, Volume 97, Issue 8, August 2006 Pages 683-688

5. Choi J., Ou J.H.J. Mechanisms of Liver Injury. III. Oxidative stress in the pathogenesis of hepatitis C virus, Am J of Phys, May 2006, 290 (5), 847-851

6. Sanjiv K. Jain, Philip W. Pemberton, Alexander Smith, Raymond F.T. McMahon et al. Oxidative stress in chronic hepatitis C: not just a feature of late stage disease, Journal of hepatology Volume 36 , Issue 6, Pages 805-811

7. Michiari Okuda, Kui Li, Michael R. Beard, Lori A. Showalter, Frank Scholle, Stanley M. Lemon, Steven A. Weinman, Mitochondrial 
injury, oxidative stress, and antioxidant gene expression are induced by hepatitis C virus core protein, Gastroenterology, 2002; 122:366-375

8. Abdalla M.Y., Ahmad I.M., Spity D.R., et al. Hepatitis C virus-core and nonstructural proteins lead to different effects on cellular antioxidant defenses. J Med Virol. 2005; 76: 489-497

9. Bureau C., Bernard J., Chaouche N., et al. Nonstructral 3 protein hepatitis $C$ virus triggers an oxidative burst in human monocytes via activation of NADPH oxidase. J Biol Chem. 2001; 276: 23077-23083

10. Görenek Levent, Acar Ali, Aydın Ahmet, Eyigun Can Polat, Çetinkaya Aytaç, Eken Ayşe and Sayal Ahmet, Oxidative stress and antioxidant defense in patients with chronic hepatitis $C$ patients before and after pegylated interferon alfa- $2 \mathrm{~b}$ plus ribavirin therapy, Journal of Translational Medicine20064:25 DOI: 10.1186/1479-5876-4-25

11. Moriya K., Nakgawa K., Santa T., et al. Oxidative stress in the absence of inflammation in a mouse model for hepatitis $\mathrm{C}$ virusassociated hepatocarcinogenesis. Cancer Res. 2001; 61: 4365-4370
12. Okuda M., Li K., Beard M.R., et al. Mithocondrial injury, oxidative stress, and antioxidant gene expression are induced by hepatitis $C$ virus core protein. Gastroenterology. 2002; 122: 366-375)

13. Tel'nykh luV., Maevskaia M.V., Glushenkov D.V. The use of hepatoprotector LiverPro in combined therapy of chronic viral hepatitis C, Klin Med (Mosk). 2008;86(11):60-2)

14. Lenard I. Stresul oxidativ în infecția cu virusul hepatitei $C$, Teză de doctorat, Conducător ştiințific Prof. Dr. Spineanu R, UMF Oradea, 2008). 900

15. Mirelle S. Fariasa, Patrícia Budnia, Carine M. Ribeiroa, Eduardo B. Parisottob, Carla Eliete I. Santosc et al. Antioxidant supplementation attenuates oxidative stress in chronic hepatitis $C$ patients Gastroenterología y Hepatologia, Volume 35, Issue 6, Pages 386-394 